\title{
Shoulder function and anatomy in complete obstetric brachial plexus palsy: long-term improvement after triangle tilt surgery
}

\author{
Rahul K. Nath • Priyanka Karicherla • \\ Faiz Mahmooduddin
}

Received: 30 April 2010 /Accepted: 3 May 2010/Published online: 16 May 2010

(C) The Author(s) 2010. This article is published with open access at Springerlink.com

\begin{abstract}
Purpose Untreated complete obstetric brachial plexus injury (COBPI) usually results in limited spontaneous recovery of shoulder function. Older methods used to treat COBPI have had questionable success, with very few studies being published. The purpose of the current study was to examine the results of triangle tilt surgery on shoulder function and development in COBPI individuals.

Methods This study was conducted as a retrospective chart review. Inclusion criteria were COBPI patients that had undergone the triangle tilt procedure from 2005 to 2009 and were between the ages of 9 months and 12 years. COBPI was defined as permanent injury to all five nerve roots (C5T1), with significant degradation in development and function of the hand. Twenty-five patients with a mean age of $5(0.75-12)$ years were followed up clinically for more than 2 years.

Results The triangle tilt procedure resulted in demonstrable clinical enhancements with appreciable improvements in shoulder function, glenoid version, and humeral head congruity. There was a significant increase in the overall Mallet score $(2.4$ points, $p<0.0001)$ following surgical correction in patients that were followed up for more than 2 years.

Conclusions The results of this study demonstrate that COBPI patients who develop SHEAR and medial rotation
\end{abstract}

R. K. Nath · P. Karicherla · F. Mahmooduddin

Texas Nerve and Paralysis Institute,

Houston, TX, USA

R. K. Nath $(\bowtie)$

6400 Fannin Street,

Suite 2420 ,

Houston, TX 77030, USA

e-mail: drnath@drnathmedical.com contracture deformities can benefit from the triangle tilt surgery, which improves shoulder function and anatomy across a range of pediatric ages. Despite these patients presenting late for surgery in general (5 years), significant improvements were observed in their glenohumeral (GH) dysplasia and their ability to perform shoulder and arm movements following surgery.

Keywords Glenoid · Humeral head · Glenohumeral dysplasia $\cdot$ SHEAR deformity $\cdot$ Medial rotation contracture (MRC) - Acromioclavicular triangle/plane (ACT/ACP) . Glenoid version $\cdot$ Posterior subluxation

\section{Introduction}

Obstetric brachial plexus injury (OBPI) is a nerve complication that usually occurs during the delivery process [1]. There is a relatively stable incidence of OBPI in the United States with 0.4 to 4.6 injuries per 1,000 live births being reported [2]. A notable proportion of cases tend to retain persistent limb deficits [3]. The most frequent nerve injury occurs in the upper $\mathrm{C} 5-\mathrm{C} 6$ roots of the brachial plexus (Erb's palsy) [4]. In this group of patients, early surgical intervention has led to improved limb function [5]. In a more devastating form of OBPI termed as total or complete OBPI (COBPI), the entire plexus, involving the upper, middle, and lower roots (C5-T1), is injured. COBPI accounts for up to $20 \%$ of all OBPI cases and often renders the individual severely compromised in arm function and growth [6].

Pediatric neurosurgeons are usually the first or among the first specialists to be consulted regarding OBPI infants. For this reason, the neurosurgeon may find interest in the indications for the surgical technique discussed in this 
paper and its results. This study will examine whether COBPI patients who develop SHEAR and medial rotation contracture deformities, both of which are discussed below, can benefit from the triangle tilt surgery. Possible management protocols at birth, as well as evaluation and treatment of older children will both be discussed.

The clinical course of these patients can, to a degree, be predicted based on their clinical presentation at birth. This is briefly summarized in Table 1. Significant function of the affected muscles will return in majority of the OBPI cases. However, the combination of rapid limb growth along with the asymmetry in the degrees of nerve involvement can lead to secondary muscle imbalances and contractures. Twisting bony deformities and loss of range of motion can develop as a result of the relatively unopposed forces of the muscles that are unaffected versus those that are denervated. This can be a vicious cycle as the bony deformities further decrease muscle and shoulder function. Muscle imbalances also arise because the denervated muscles do not grow at the same rate as their functional, opposing muscles. Later, as the nerves heal, muscles regain function in an unbalanced pattern. Co-contractions and contractures can develop in the abductors and external rotators versus the adductors and internal rotators, as well as the biceps versus the triceps. In both cases, as the unaffected muscle group(s) contract, they are unopposed. This creates a tethering effect that limits passive range of motion of the unbalanced muscles, which leads to the typical arm position seen in OBPI children: abduction and medial rotation at shoulder, forearm pronation, and flexion at elbow [7]. In addition to the asymmetric muscle action in the shoulder mentioned above, bony deformities can also develop as a result of reduced innervation to affected bones [8-11]. Eventually, muscular imbalances around the shoulder joint can lead to progressive GH dysplasia and instability [12]. Often accompanying changes to the glenoid, progressive subluxation of the humeral head can also be observed. Associated with the type and severity of these deformities are both age and degree of medial rotation contracture, which is discussed below. The SHEAR deformity can ultimately develop as well, which is also discussed in further detail below.
Although most unoperated children do recover some function, albeit much impaired, patients with COBPI are at an elevated risk of developing a dysfunctional limb [13]. These patients usually present with severe medial rotation contracture (MRC). MRC is an internal rotation contracture caused by a constant downward pressure exerted by the acromion on the humeral head. In a normal shoulder girdle at rest, the acromion should not articulate with the humeral head. However, in many OBPI patients, one of the major bony deformities seen is the extension and elevation of the scapula above the clavicle and is termed as the SHEAR (scapular hypoplasia, elevation, and rotation) deformity. The SHEAR deformity is responsible for tilting of the acromioclavicular joint from a neutral position, which in turn displaces the humeral head from the glenoid fossa (Figs. 1, 2) [4, 14]. With the severe MRC, COBPI patients also usually present with poor shoulder movements and inadequate hand function [2]. In addition to presenting with classic OBPI features such as posterior subluxation of the humeral head, scapular elevation, and glenoid version, these individuals also bear deformities such as elbow angulation (secondary to an abducted and medially rotated shoulder), supination disability (due to the C6 injury weakening the biceps muscle, the major supinator of the forearm), and shortened arms.

Limited literature is offered on the assessment of surgical outcomes on shoulder deformities for patients with C5-T1 injuries, reflecting the traditional lack of availability of effective surgical options. The current study was conducted in order to analyze the results from the triangle tilt procedure in pediatric individuals with COBPI by reviewing their computed tomography reports as well as by evaluating their shoulder functions before and after surgery $[4,15]$. Conceptually, the triangle tilt is a bony surgical procedure that detaches the distal acromioclavicular triangle ("ACT", with its sides defined by the clavicular shaft and the acromion process and its base by an imaginary line connecting their medial ends)-humeral head complex from the abnormally positioned scapula; subsequent reversal of the anterior tilt of the ACT then would allow a natural rotation of the humeral head back into a more neutral

Table 1 Narakas classification of brachial plexus injury based on clinical observations and expected typical recoveries

\begin{tabular}{lll}
\hline Group & Newborn clinical presentation & Typical Recovery \\
\hline 1 & $\begin{array}{c}\text { Absence of abduction, external rotation, elbow } \\
\text { flexion, forearm supination }\end{array}$ & Recovery begins within 1 month; full recovery by 4-6 months \\
2 & $\begin{array}{c}\text { Similar to group 1 with weaker active elbow } \\
\text { extension }\end{array}$ & $\begin{array}{c}\text { 1 month before recovery if shoulder and elbow flexion, steady improvement } \\
\text { but likely development of contractures that limit motion }\end{array}$ \\
3 & $\begin{array}{l}\text { Fail shoulder, absence of elbow flexion, weak } \\
\text { extension, flexed wrist, closed fist }\end{array}$ & $\begin{array}{c}\text { Slow partial recovery of up to 15 months; weak, limited function and secondary } \\
\text { deformities likely }\end{array}$ \\
4 & $\begin{array}{c}\text { Flail extremity, half-open hand, little finger } \\
\text { movement }\end{array}$ & $\begin{array}{c}\text { Poor shoulder function; arm rests in abduction, elbow flexion, internal rotation, } \\
\text { supinated forearm; absence of external rotation }\end{array}$ \\
\hline
\end{tabular}


position within the glenoid fossa $[4,7,15]$. This procedure differs from more conventional approaches (tendon transfers, axillary nerve decompressions, and external rotation osteotomy of the humerus) in that it addresses and corrects the scapular hypoplasia, elevation and external rotation (SHEAR) deformity associated with typical OBPI cases [14].

All patients had undergone the modified Quad procedure prior to triangle tilt surgery at the same institution [16]. The modified Quad is a modification of the Quad Procedure, which is a soft tissue release operation performed in patients with partially recovered brachial plexus injuries to improve shoulder abduction and flexion function. The Quad couples neurolysis and decompression of the axillary nerve with an untethering release of soft tissue contractures that limit abduction power [7]. The modified Quad is a modification of the combination of muscles released and their inset positions to improve upon a previously described operation by Narakas [17].

More specifically, it is based on the following four steps:

(1) Latissimus dorsi muscle release and transfer for external rotation and abduction.

(2) Teres major muscle release and transfer for scapular stabilization.

(3) Subscapularis release without transfer.

(4) Axillary nerve decompression and neurolysis.

Other nerve decompressions or muscle/tendon transfers (such as pectoralis muscle releases) might be performed at the same time, depending on the individual child. In the modified Quad procedure, the latissimus dorsi, teres major, subscapularis, and pectoralis muscle contractures are released [7]. As performed by Narakas, the latissimus dorsi

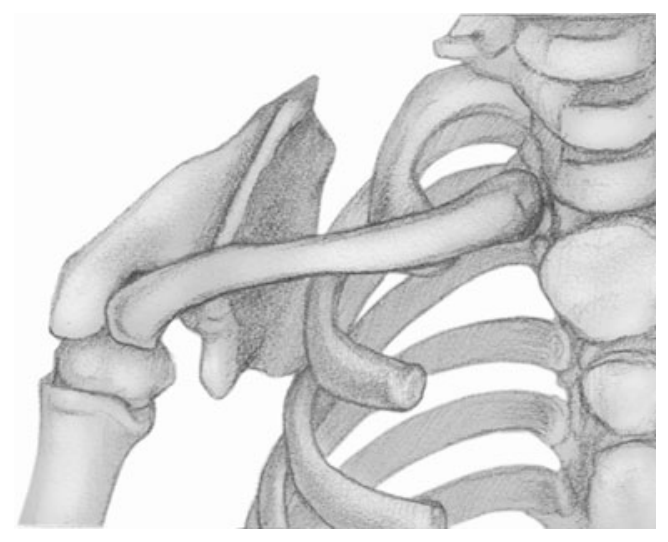

Fig. 1 Pre-triangle tilt acromioclavicular plane in a shoulder with SHEAR deformity. Notice the abnormal position of the ACT (sides defined by clavicular shaft and acromion process and base as an imaginary line connecting their medial ends) in relation to the humeral head. The ACT lies in the acromioclavicular plane and teres major muscles are sutured to a low position in the teres minor muscle [17]. The deltoid can now act more effectively, due to the enhanced stabilization of the rotator cuff, while not tethering shoulder abduction and flexion ability [7].

This study will examine the surgical outcomes and results of the triangle tilt procedure in COBPI patients with shoulder deformities in order to determine if it is an effective surgical option that leads to significant improvements in shoulder and arm function and anatomy.

\section{Methods}

\section{Patients}

This study was conducted as a retrospective chart review. Inclusion criteria were COBPI patients that had undergone the triangle tilt procedure between 2005 and 2009 and were between the ages of 9 months and 12 years old. Exclusion criteria were patients who had only partial OBPI, those who did not have triangle tilt, and ones younger than 9 months or older than 12 years. Twenty-five patients were followed up clinically for more than 2 years. The patient population was comprised of 16 boys and 9 girls with an age range of 9 months to 12 years (mean age $=5$ years) at the time of the triangle tilt operation. In addition to all patients having had the modified Quad prior to triangle tilt at the same institution, other operations some of the patients had prior to triangle tilt included posterior glenohumeral capsulorrhaphy $(N=5)$, biceps tendon lengthening $(N=7)$, forearm osteotomy $(N=4)$, and wrist capsulodesis $(N=1)$.

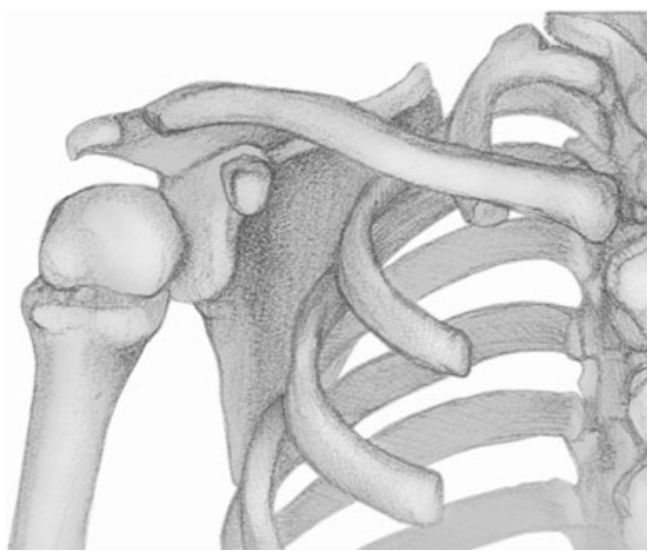

Fig. 2 Neutral anatomic acromioclavicular plane. Notice the separation of the distal ACT-humeral head complex from the normally positioned scapula. Post-triangle tilt, the reversal of the anterior tilt of the ACT allows a natural rotation of the humeral head back into a more neutral position within the glenoid fossa 
Surgical technique

The triangle tilt procedure was developed by the senior author (RKN). The procedure involves tilting of the acromioclavicular plane (in which the ACT lies) to a neutral and functional position through osteotomies of the clavicle, acromion process and scapula, thereby allowing for the repositioning of the humeral head into the glenoid fossa, in a more anatomic situation. See Fig. 1 for a preoperative plane, versus Fig. 2, a neutral anatomic plane.

More specifically, the triangle tilt surgery consists of:

(1) osteotomy of the clavicle at the junction of the middle and distal thirds

(2) osteotomy of the acromion process at its junction with the spine of the scapula

(3) ostectomy of the superomedial angle of the scapula to reduce scapular winging

(4) splinting of the extremity in adduction, external rotation, and forearm supination

Additional minor elements of the procedure include bone grafting of the acromion process and clavicular osteotomy sites and semi-rigid fixation of the clavicular osteotomy segments to prevent nonunion [7]. See Figs. 3 and 4 for illustrations of the procedure and the bone realignment following triangle tilt surgery.

Evaluation of patients through assessment of bony deformities

All clinical evaluations and measurements were made by trained research scientists, independent of the surgeon and senior author. These scientists were trained in assessing glenohumeral deformity qualitatively by clinical examination and quantitatively through measurements from computed tomography (CT) scans and 3D reconstructions as described previously [4].

Glenoid version was calculated as described by Friedman et al. [18]. A scapular line connecting the medial margin of the scapula to the middle of the glenoid was drawn at the mid-glenoid level. The glenoscapular angle, which is defined as the angle formed between the scapular line and a line drawn lateral to the glenoid surface closely interacting with the humeral head, was measured and $90^{\circ}$ was subtracted from it to evaluate glenoid version. A value of 0 is considered normal, but some variation is possible (mean: $2 \pm 5$ degrees of anteversion, range, $14^{\circ}$ of anteversion to $12^{\circ}$ of retroversion). The percentage of humeral head subluxation was calculated by taking the ratio of the greatest diameter of the humeral head divided by the perpendicular distance between the anterior portion of humeral head and scapular line and then multiplied by 100. A value of 50 is considered normal. See Fig. 5 for a schematic drawing showing the method of calculating the glenoscapular angle, glenoid version $(\theta)$, and posterior subluxation of the humeral head.

In order to assess SHEAR deformity, also referred to as scapular elevation, the anterior view of the $3 \mathrm{D}$ reconstruction was used to measure the area of the scapula visible over the clavicle through a Universal Desktop Ruler (AVP Soft, version.2.8.1112, Voronezh, Russia) and then divided by the total area of the scapula in the affected shoulder. The scapular elevation in the contralateral shoulder was then subtracted from that in the affected shoulder, and this value was multiplied with 100 to express SHEAR as a percentage. A value of 0 is considered normal.

Clinical evaluation of patients by shoulder function

Patients were evaluated preoperatively and postoperatively through video recordings using a modified Mallet scale [19] (Fig. 6). Depending on their ability to perform certain shoulder and arm movements (position of arm at rest, abduction, external rotation, hand-to-mouth, hand-to-neck, hand-to-spine, and supination), patients were scored on a scale of $1-5$, with 1 being most affected and 5 being normal. The overall Mallet score (5-25) is calculated based on five of these movements, with supination being expressed as a degree measurement. In young children ( $<2$ years old), shoulder function can be assessed by holding a toy or lollipop or placing a sticker on a part of the child's body and having the child reach for it. Only four patients in this study were less than 2 years old. All of these evaluations were performed by trained research scientists, independent of the senior author.

\section{Statistical analyses}

The mean and standard error mean were calculated from patient scores using Microsoft Excel. The comparison of mean values for each parameter was determined using the paired, two-tailed, Student's $t$ test with Analyse-it software (Leeds, United Kingdom) for Microsoft Excel. A two-tailed $p$ value of $<0.05$ was considered statistically significant.

\section{Results}

The patient demographics and outcomes of triangle tilt surgery are depicted in Tables 2, 3 and Figs. 7, 8, 9 and 10. All patients initially presented with severe medial rotation contracture and limited shoulder function.

The latest follow-up examinations (mean=27 months) showed that the overall Mallet score increased significantly from 12 to 14.6 points $(p<0.0001$, Table 3$)$. All patients had undergone modified Quad surgery prior to triangle tilt 
Fig. 3 Artist's rendering of the triangle tilt surgery and anterior capsule release. Illustrated are osteotomies of the clavicle, scapula, and acromion process, along with glenohumeral capsulodesis and anterior capsule release

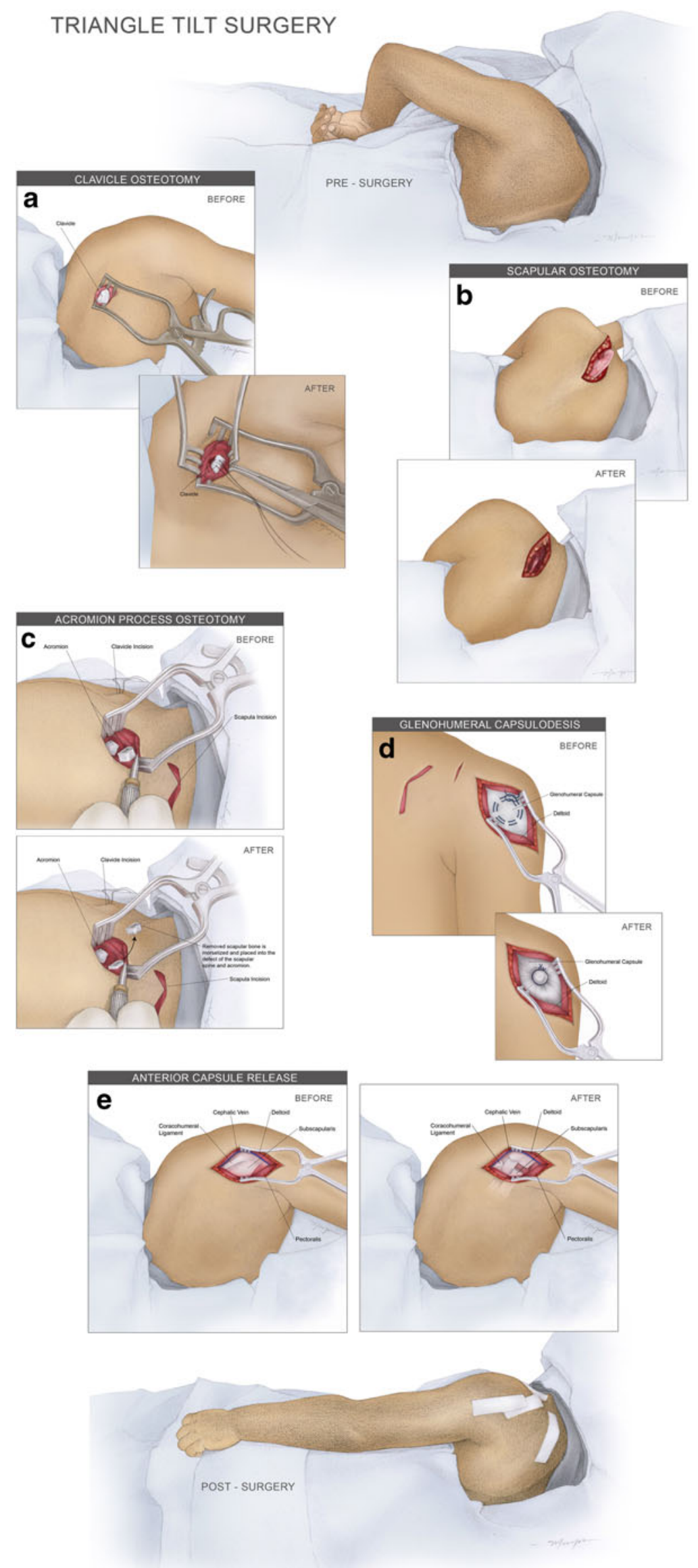


Fig. 4 Artist's rendering of acromioclavicular interface before and after triangle tilt surgery. Notice the bone realignment following triangle tilt surgery

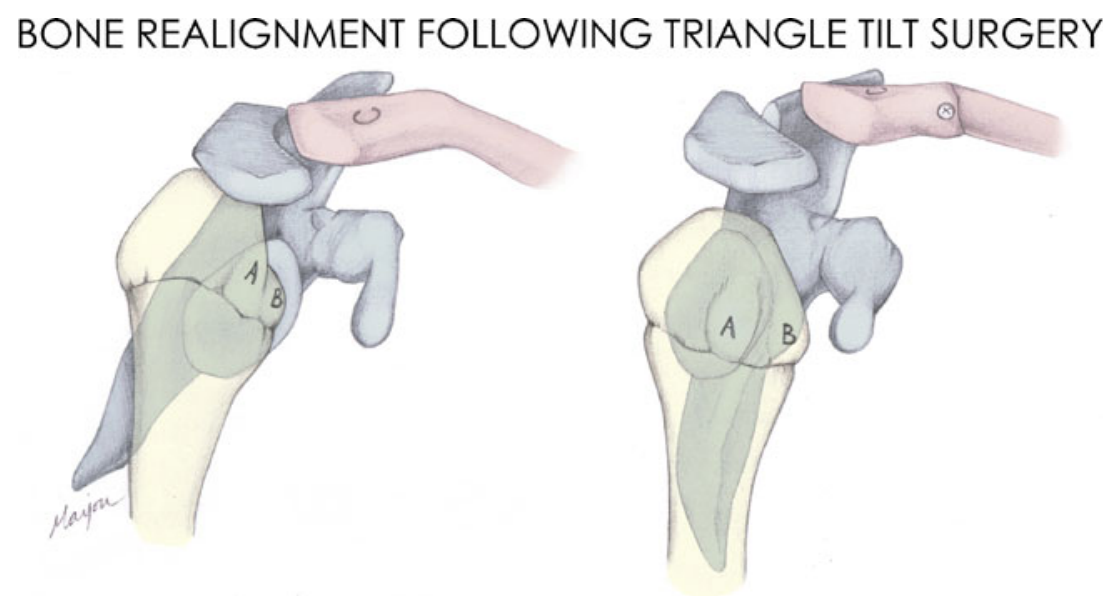

and therefore, did not exhibit considerable changes in abduction $(p=0.41)$. It must be mentioned that improvement in global abduction following modified Quad surgery was seen in all patients (data not shown) and has previously been demonstrated in other OBPI patients by the same surgeon [16]. The patients displayed significantly improved hand-to-mouth $(p<0.0001)$, hand-to-neck $(p \leq 0.0001)$, and hand-to-spine $(p<0.05)$ functions (Table 3, Fig. 9). Forearm supination improved by a mean of $33.2^{\circ}$ postoperatively $(p<0.05)$. Significant improvements were also noted in external rotation $(p<0.0001)$ and the posture of the arm at rest (Fig. 10). All patients, regardless of their age (0.7512 years), demonstrated significant improvements in shoulder function. No differences were observed in the overall improvement in Mallet scores between younger $(\leq 4$ years, $N=12$ ) and older $(>4$ years, $N=13)$ pediatric patients $(p>$ $0.05)$, with both groups equally benefiting from the surgery.

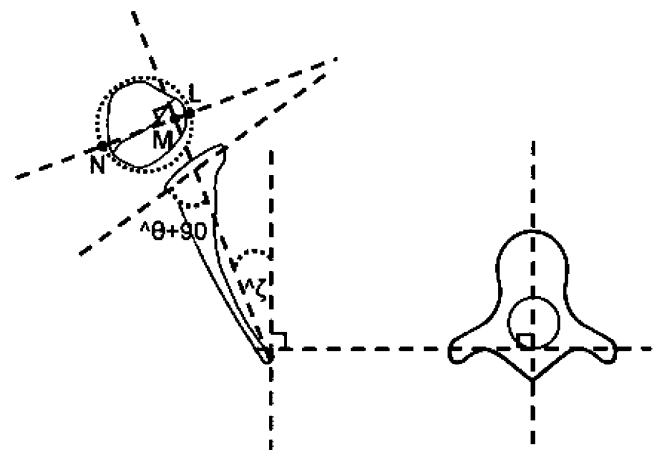

Fig. 5 Schematic drawing showing the method of calculating glenoscapular angle (glenoid version $\theta$ ) and posterior subluxation of the humeral head. The scapular line that connects the medial aspect of the scapula and the mid-glenoid is drawn. A second line is drawn connecting the posterior and anterior margins of the glenoid. $90^{\circ}$ is subtracted from the angle of the posterior medial quadrant defined by these lines to determine the glenoid version $\theta$. A line perpendicular to the scapular line is drawn and the percentage of posterior subluxation is defined as the ratio of the distance from the scapular line to the anterior portion of the head to the diameter of the humeral head (LM/ $\mathrm{LN} \times 100)$
Measurements taken from axial CT images (Fig. 7) and 3D-reconstructions (Fig. 8) demonstrated the improvements in posterior subluxation from $13 \%$ preoperatively to $30.4 \%$ postoperatively $(p<0.05)$ and glenoid version from $-28^{\circ}$ preoperatively to $-16.5^{\circ}(p<0.05)$ following triangle tilt surgery. There was lesser elevation of the affected scapulae, decreasing the SHEAR deformity from $6.4 \%$ to $2.9 \%(p<$ $0.05)$. Taken together, these results demonstrate the effectiveness of triangle tilt surgery in improving shoulder function in this study group.

\section{Discussion}

The most frequent, persistent complications of OBPIs are anatomical and functional deformities of the affected shoulder [20]. In cases of COBPI, a lack of spontaneous recovery by the age of 3 months results in an increasingly poor prognosis for shoulder development [21]. For this reason, the modified Quad can be performed as early as 6 months of age if there is no spontaneous recovery and the clinical condition warrants it (presence of muscle contractures, limitation of shoulder range of motion, especially abduction). This can then be followed by the triangle tilt as early as 3 months later (age of 9 months) to correct the SHEAR deformity. Most of our patients were operated on at a mean age of 5 years either because they did not seek surgical help earlier or had undergone unsuccessful operations at outside institutions. In one case, the patient had undergone 11 previous operations at another institution, some of which worsened his condition.

Early treatment may entail physical and occupational therapy, daily passive range of motion exercises, BTX-A injections, and/or splinting to lessen the severity of biceps/ triceps co-contraction [22, 23]. Many times, however, deformations occur that require surgical intervention. For the best outcomes, tightness in the axilla and pectoralis muscles must be treated early and aggressively. There are 


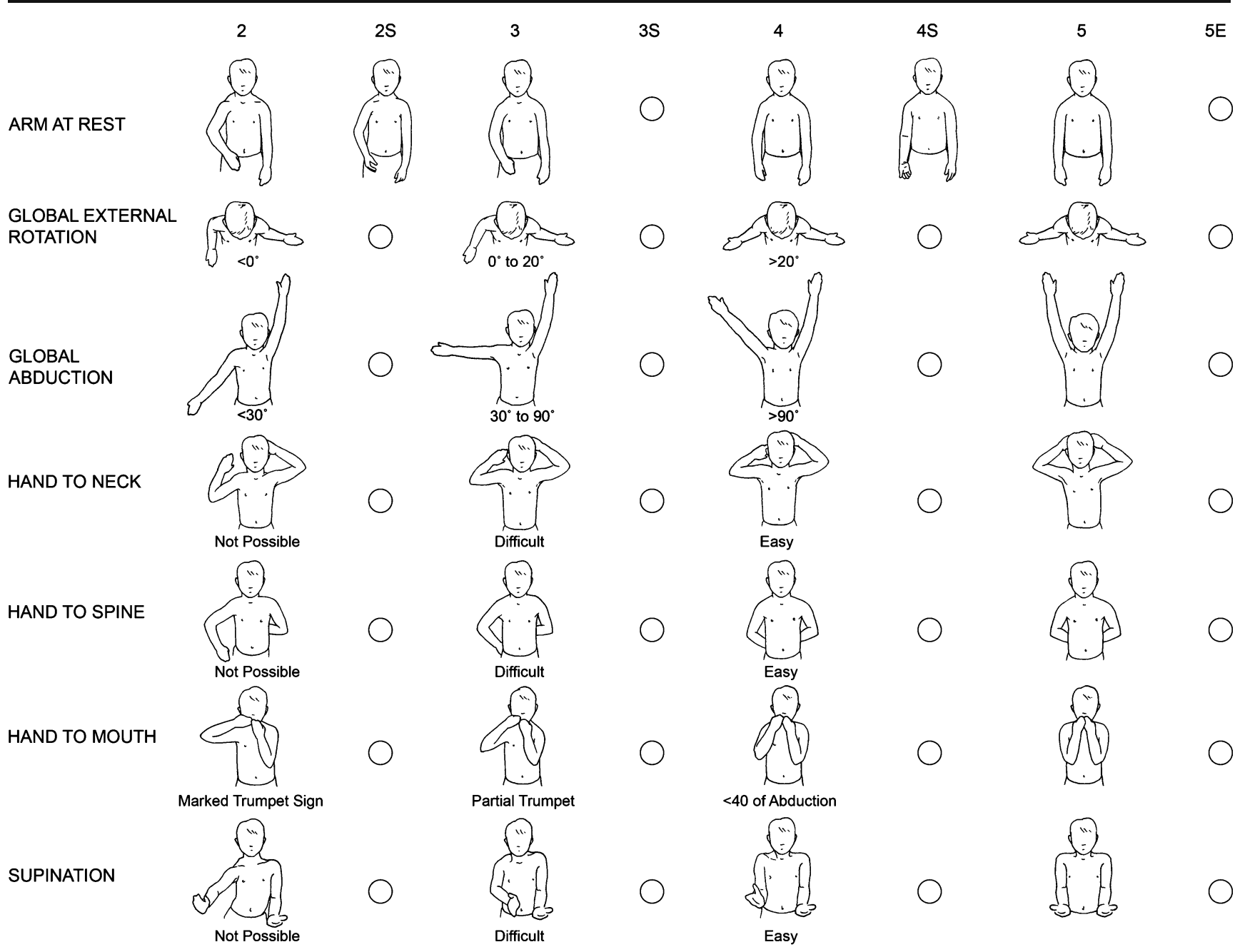

Fig. 6 The Nath Modification of Mallet's System: clinical scoring of function. In addition to assessing the classical functions of the Modified Mallet system, supination and the resting position are

no studies that show any benefit of therapy alone or in combination with Botulinum toxin or splinting when fixed axillary and chest contractures or medial rotation contracture of the shoulder occur. These are structural musculoskeletal deformities that are best treated surgically, according to the scientific and medical literature. The modified Quad is an effective soft tissue procedure to improve the abduction deficit. The medial rotation contracture of the shoulder due to SHEAR deformity has been more difficult to correct [7]. Traditionally, the derotational humeral osteotomy has been used to improve the position of the hand and forearm [24-27]. Although humeral osteotomy may provide a level of functional improvement, the procedure does not address the GH deformity or aim to correct shoulder dynamics. It places the arm in a more functional position but does not account for the presence of SHEAR deformity and its resultant motion-limiting impingement. Further, humeral osteotomy has a high failure rate and does not correct or attempt to correct the $\mathrm{GH}$ evaluated. To further define deformity, fixed forearm supination (positions $2 \mathrm{~S}, 3 \mathrm{~S}$, and $4 \mathrm{~S}$ ) as well as external rotation position (5E) are scored

deformities associated with SHEAR and OBPI. Another surgical procedure, the anterior GH capsule release, rotates the humerus within an abnormal glenoid without attempting to address the abnormal structure and angles of the scapula and glenoid fossa. This carries a high risk of unsatisfactory external rotation deformity, which can be difficult to treat and may be functionally more limiting than the medial rotation contracture the procedure is designed to address [7]. The goal of GH centralization, however, is valid and seems to result in improved glenoid anatomy following normalization of the humeral head within the fossa [28]. Another surgical option, posterior GH capsulorrhaphy, tightens the posterior capsule surrounding the humeral head and repositions it anteriorly. However, it also fails to take into account the SHEAR deformity and its central influence in the pathophysiology of the medial rotation contracture. In the senior author's experience, posterior capsulorrhaphy has a high failure rate when used on its own [7]. The triangle tilt operation addresses the pathophysiology of the 
Table 2 Patient demographics

$T T$ triangle tilt, $M Q$ modified Quad, $P G H C$ posterior glenohumeral capsulorrhaphy, $B T L$ biceps tendon lengthening, $\mathrm{HO}$ humeral osteotomy, $F O$ forearm osteotomy, $W C$ wrist capsulodesis MQ was performed prior to TT in all patients

\begin{tabular}{|c|c|c|c|c|c|}
\hline Patient & Sex & Follow up (month) & TT date & Age at TT (yr.mo.) & Other surgeries before TT \\
\hline 1 & M & 25 & $9 / 11 / 2007$ & 12 & MQ, PGHC1,BTL,HO \\
\hline 2 & M & 14 & $4 / 19 / 2006$ & 7.7 & MQ, PGHC1, BTL \\
\hline 3 & M & 34 & $9 / 27 / 2006$ & 6.9 & MQ, BTL \\
\hline 4 & M & 32 & $8 / 16 / 2006$ & 3.1 & MQ, FO \\
\hline 5 & M & 26 & $5 / 9 / 2007$ & 9.1 & MQ, HO, N,WC \\
\hline 6 & M & 21 & $11 / 16 / 2006$ & 2.7 & MQ \\
\hline 7 & $\mathrm{~F}$ & 28 & $11 / 10 / 2005$ & 3.1 & MQ \\
\hline 8 & M & 20 & $6 / 26 / 2007$ & 2.8 & MQ \\
\hline 9 & M & 50 & $5 / 10 / 2005$ & 6.1 & MQ \\
\hline 10 & M & 33 & $3 / 1 / 2006$ & 5.2 & MQ,PGHC1,HO,FO, \\
\hline 11 & $\mathrm{~F}$ & 53 & $2 / 17 / 2005$ & 7.9 & MQ,PGHC1,BTL,FO,HO \\
\hline 12 & $\mathrm{~F}$ & 20 & $8 / 28 / 2007$ & 3.9 & MQ \\
\hline 13 & $\mathrm{~F}$ & 38 & $7 / 19 / 2005$ & 5.1 & MQ,BTL \\
\hline 14 & M & 12 & $9 / 23 / 2008$ & 4.4 & MQ \\
\hline 15 & $\mathrm{~F}$ & 30 & $5 / 2 / 2007$ & 2.9 & MQ \\
\hline 16 & M & 20 & $6 / 15 / 2006$ & 10.1 & MQ, BTL \\
\hline 17 & M & 38 & $7 / 21 / 2005$ & 3.7 & MQ, FO, HO \\
\hline 18 & $\mathrm{~F}$ & 15 & $6 / 21 / 2006$ & 1.4 & MQ \\
\hline 19 & M & 15 & $6 / 5 / 2008$ & 1.3 & MQ \\
\hline 20 & $\mathrm{~F}$ & 19 & $8 / 11 / 2006$ & 11.8 & MQ, PGHC1 \\
\hline 21 & $\mathrm{~F}$ & 19 & $1 / 4 / 2007$ & 2.8 & MQ \\
\hline 22 & M & 36 & $8 / 18 / 2005$ & 4.3 & MQ \\
\hline 23 & M & 32 & $3 / 15 / 2006$ & 4.5 & MQ \\
\hline 24 & $\mathrm{~F}$ & 35 & $11 / 15 / 2006$ & 1.9 & MQ \\
\hline \multirow[t]{2}{*}{25} & M & 11 & $7 / 17 / 2008$ & 0.9 & MQ \\
\hline & & Avg. $=27$ months & & Avg. $=5$ years & \\
\hline
\end{tabular}

Table 3 Comparison of modified Mallet scores and SHEAR deformity parameters before and after triangle tilt surgery in patients with C5-T1 injury

\begin{tabular}{|c|c|c|c|c|}
\hline Modified mallet functions scoring scale $=1-5$ & $\begin{array}{l}\text { Pre-triangle tilt } \\
\text { Mean } \pm \text { SEM }\end{array}$ & Post-triangle tilt & Difference in mean values & Significance \\
\hline Abduction & $3.9 \pm 0.1$ & $3.8 \pm 0.1$ & 0.1 & $p=0.41$ \\
\hline Hand-to-mouth & $1.7 \pm 0.7$ & $2.7 \pm 0.2$ & 1 & $p<0.0001^{*}$ \\
\hline Hand-to-neck & $2.2 \pm 0.6$ & $2.7 \pm 0.7$ & 0.1 & $p<0.001 *$ \\
\hline Hand-to-spine & $2 \pm 0.1$ & $2.3 \pm 0.1$ & 0.3 & $p=0.016^{*}$ \\
\hline External rotation & $2.2 \pm 0.1$ & $2.9 \pm 0.2$ & 0.7 & $p<0.0001^{*}$ \\
\hline Supination $\left({ }^{\circ}\right)$ & $-37 \pm 7$ & $-2 \pm 8$ & 34.4 & $p=0.001^{*}$ \\
\hline Overall Mallet score & $11.9 \pm 0.4$ & $14.4 \pm 0.4$ & 2.5 & $p<0.0001^{*}$ \\
\hline \multicolumn{5}{|l|}{ SHEAR deformity } \\
\hline Posterior subluxation (\%) & $13 \pm 7.3$ & $30.4 \pm 4.5$ & 17.5 & $p=0.01 *$ \\
\hline Glenoid version $\left({ }^{\circ}\right)$ & $-28 \pm 5$ & $-16.5 \pm 4$ & 11.5 & $p=0.0026^{*}$ \\
\hline Scapular elevation $(\%)$ & $6.4 \pm 2$ & $2.9 \pm 0.1$ & 3.6 & $p=0.045^{*}$ \\
\hline
\end{tabular}

Angle of apparent active supination was recorded as follows: $0^{\circ}=$ neutral position, $90^{\circ}=$ full apparent supination, $-90^{\circ}=$ full apparent pronation *Statistically significant difference $(p<0.05)$ 
Fig. 7 Axial CT images of a complete plexus injury patient illustrating a humeral head posterior subluxation preceding surgery and $\mathbf{b}$ corrective repositioning of the humeral head post-surgery. $R$ indicates right, $L$ indicates left, $H$ indicates humeral head, $G$ indicates glenoid a

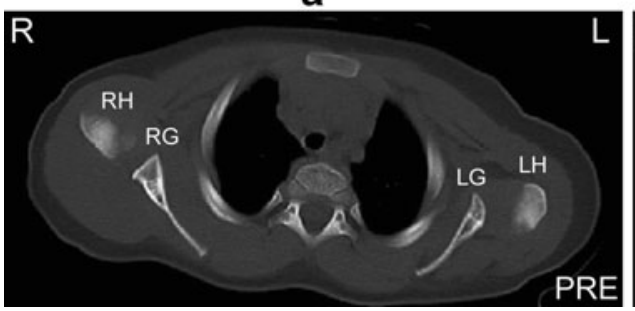

b

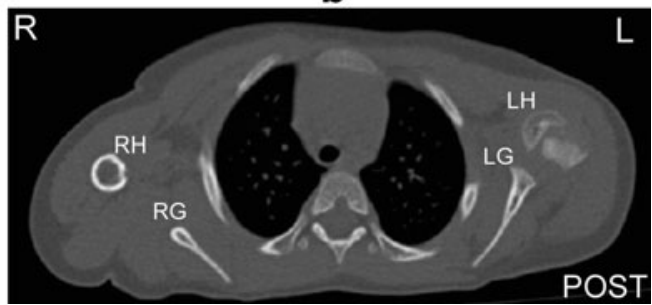

medial rotation contracture directly and has been shown to improve function, anatomy, and development of the shoulder joint in OBPI patients [4, 14-16, 19, 29]. The efficacy of the triangle tilt surgery has been shown in the current study to extend to COBPI patients. Conventional management has involved muscle releases and nerve transfers to try and correct glenohumeral dysplasia in COBPI patients [2, 30]. Our experience strongly suggests that modified Quad and triangle tilt surgeries in combination offer superior functional outcomes to nerve repair alone. Further, modified Quad and triangle tilt surgeries provide superior functional outcomes compared to nerve grafting with or without ancillary secondary operations [29].

In summary, we provide recommendations regarding the management of COBPI patients including possible management options immediately after birth, as well as when older children are seen. If a child is diagnosed with COBPI at birth and referred to appropriate medical care early, the recommendation is to follow the patient for at least 6 months to monitor for any signs of clinical improvement. During this time, conservative management including physical and occupational therapy, daily passive range of motion exercises, BTX-A injections, and/or splinting to prevent the occurrence of biceps/triceps co-contraction, is recommended. If there is no spontaneous recovery and the clinical condition warrants it (presence of muscle contractures, limitation of shoulder range of motion, especially abduction), the modified Quad can be performed as early as 6 months, followed by the triangle tilt as early as 3 months later (age of 9 months) to correct the SHEAR deformity. In older children, the same evaluation systems would apply (CT measurements and clinical evaluation through Mallet grading). The modified Quad can actually be performed as late as into adulthood if the patient has the indications. The triangle tilt, however, is limited by the declining ability of the glenohumeral joint to remodel, typically seen around the ages of 16-17 years.

Restoration of a functional limb in total plexus palsy remains challenging owing to the complexity of the injury. Glenohumeral dysplasia leads to shoulder deformities that limit motion and interfere with the quality of life [29]. Corrective realignment of the humeral head into the glenoid is therefore necessary for restoration of shoulder function and joint remodeling [28, 31]. Although humeral osteotomy may moderately improve shoulder function [27], it does not address glenohumeral dysplasia or joint remodeling and therefore increases the possibility of recurrence [15]. One of the major bony deformities in these patients is the SHEAR deformity [4, 14]. Many groups fail to address the SHEAR deformity when trying to correct glenohumeral dysplasia. In SHEAR deformity, the distal ACT (acromion and clavicle) impinges against the humeral head due to the abnormally elevated scapula. The triangle tilt procedure eliminates the impingement caused due to SHEAR deformity through osteotomies of the clavicle and acromion,
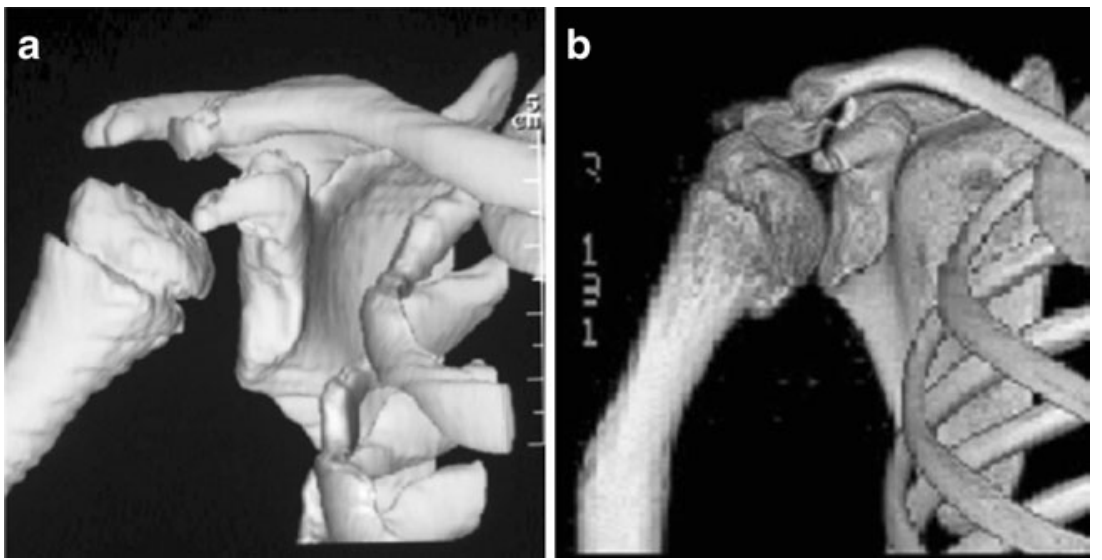

Fig. 8 3D-CT images of a complete plexus injury patient showing a preoperative impingement of the acromioclavicular triangle on the humeral head $\mathbf{b}$ postoperative release of the acromioclavicular triangle allowing for repositioning of humeral head 
Fig. 9 Shoulder functions in a patient with COBPI before $(\mathbf{a}-\mathbf{c})$ and after $(\mathbf{d}-\mathbf{f})$ triangle tilt surgery. Movements include handto-mouth (a, d), hands to spine $(\mathbf{b}, \mathbf{e})$ and hands to neck $(\mathbf{c}, \mathbf{f})$. The ability to perform all of these functions improved significantly postoperatively
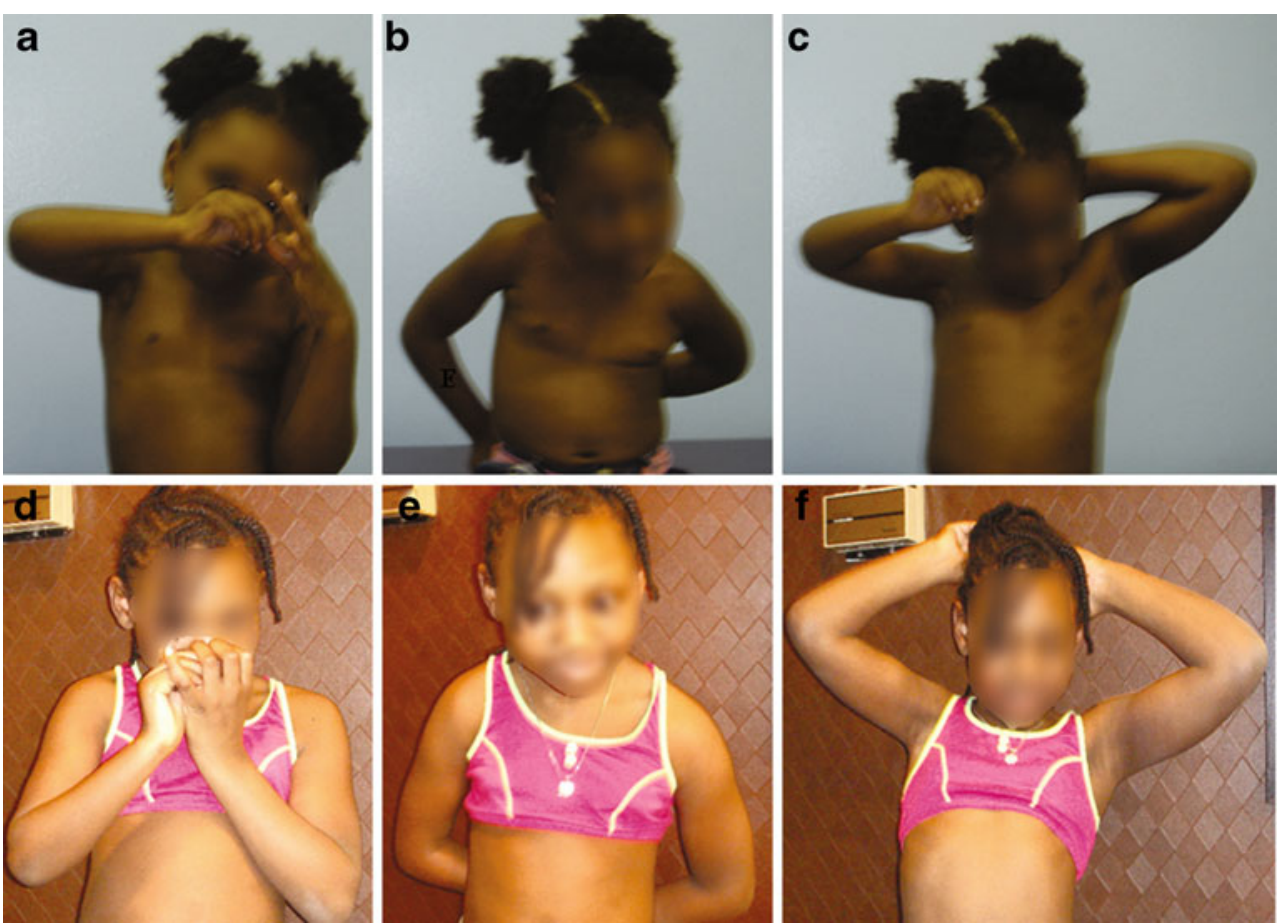

thereby allowing the humeral head to realign itself in a more normal position into the glenoid (Fig. 8) [4, 20]. As the triangle tilt procedure aims at improving the glenohumeral alignment, long-term improvement in shoulder function and joint remodeling are expected. Significant improvements in humeral head position, glenoid version, and SHEAR deformity were observed in COBPI patients following triangle tilt surgery $(p<0.05$, Table 3$)$.

Shoulder functions were assessed using the modified Mallet scale as described previously [4, 19]. There was a significant improvement in the ability of these patients to perform shoulder and arm movements, thereby increasing the overall mean Mallet score from a preoperative figure of

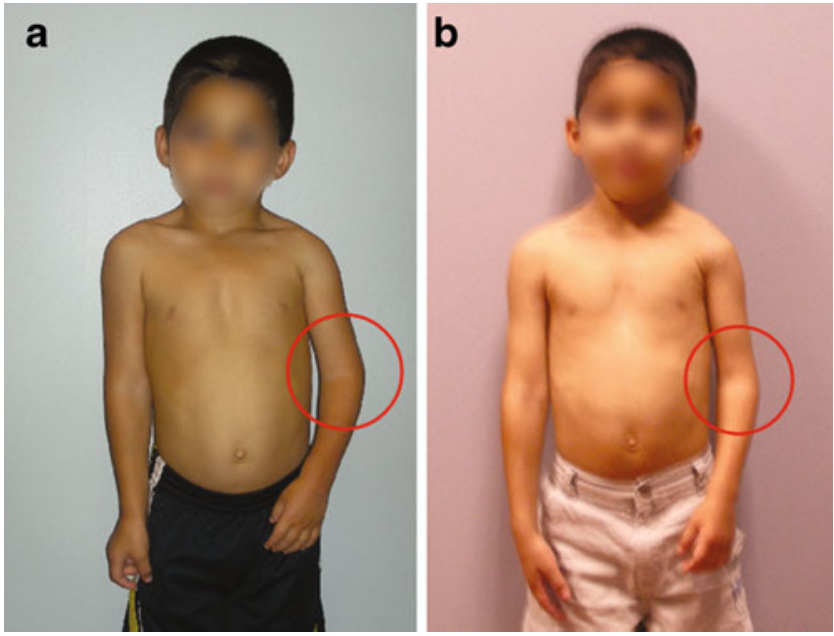

Fig. 10 Position of arm at rest a prior to triangle tilt surgery; $\mathbf{b} 1$ year post-triangle tilt
12 to a post-operative figure of 14.6 points (Table 3). The position of the arm at rest was also superior, as indicated by the reduction in elbow angulation (Fig. 10). Although all patients did not regain the ability to supinate completely, it improved by a mean of $34^{\circ}$ postoperatively. Twelve of our patients were below the age of 4 years (range $=0.75-3.9)$ and 13 of them were aged over 4 years (range $=4-12$ ) at the time of surgery. No differences were observed in the overall improvement in shoulder functions between these two groups, implying that the surgery benefited patients over a broad age range.

In conclusion, this study demonstrates that COBPI patients who develop the medial rotation contracture and SHEAR deformities may benefit from the triangle tilt surgery. The results obtained from this retrospective review were encouraging and established that the triangle tilt procedure led to significant improvements in the glenohumeral dysplasia of patients with COBPI, even though these patients in general presented late for surgery (5 years). Significant improvements were observed in the ability of these patients to perform shoulder and arm movements following surgery. The success of triangle tilt surgery in improving shoulder function and anatomy opens up a new and beneficial surgical option for total plexus palsy patients.

\section{Limitations}

Some of the patients in this study population had other operations prior to having triangle tilt surgery, including posterior glenohumeral capsulorrhaphy $(N=5)$, biceps tendon lengthening $(N=7)$, forearm osteotomy $(N=4)$, and 
wrist capsulodesis $(N=1)$. This heterogeneity should be taken into account when interpreting improvements in Mallet scores. Future studies to assess a group of COBPI patients, none of which have had any shoulder/arm surgeries prior to the triangle tilt besides the modified Quad, are encouraged.

Open Access This article is distributed under the terms of the Creative Commons Attribution Noncommercial License which permits any noncommercial use, distribution, and reproduction in any medium, provided the original author(s) and source are credited.

\section{References}

1. Pollack RN, Buchman AS, Yaffe H, Divon MY (2000) Obstetrical brachial palsy: pathogenesis, risk factors, and prevention. Clin Obstet Gynecol 43:236-246

2. Maillet M, Romana C (2009) Complete obstetric brachial plexus palsy: surgical improvement to recover a functional hand. J Child Orthop 3:101-108

3. Hoeksma AF, Ter Steeg AM, Dijkstra P, Nelissen RG, Beelen A, de Jong BA (2003) Shoulder contracture and osseous deformity in obstetrical brachial plexus injuries. J Bone Joint Surg (Am) 85A:316-322

4. Nath RK, Lyons AB, Melcher SE, Paizi M (2007) Surgical correction of the medial rotation contracture in obstetric brachial plexus palsy. J Bone Joint Surg Br 89:1638-1644

5. Price A, Tidwell M, Grossman JA (2000) Improving shoulder and elbow function in children with Erb's palsy. Semin Pediatr Neurol 7:44-51

6. Shenaq SM, Berzin E, Lee R, Laurent JP, Nath RK, Nelson MR (1998) Brachial plexus birth injuries and current management. Clin Plast Surg 25:527-536

7. Nath RK (2007) Obstetric brachial plexus injuries-Erb's palsy: the Nath method of diagnosis and treatment. VirtualBookworm.com Publishing, College Station

8. Bubenik GA, Bubenik AB, Stevens ED, Binnington AG (1982) The effect of neurogenic stimulation on the development and growth of bony tissues. J Exp Zool 219:205-216

9. Edoff K, Hellman J, Persliden J, Hildebrand C (1997) The developmental skeletal growth in the rat foot is reduced after denervation. Anat Embryol (Berl) 195:531-538

10. Garcia-Castellano JM, Diaz-Herrera P, Morcuende JA (2000) Is bone a target-tissue for the nervous system? New advances on the understanding of their interactions. Iowa Orthop J 20:49-58

11. Hukkanen M, Konttinen YT, Santavirta S, Paavolainen P, Gu XH, Terenghi G, Polak JM (1993) Rapid proliferation of calcitonin gene-related peptide-immunoreactive nerves during healing of rat tibial fracture suggests neural involvement in bone growth and remodelling. Neuroscience 54:969-979

12. Birch R, Bonney G, Wynn Parry CB (1998) Birth lesions of the brachial plexus. In: Birch R, Bonney G, Wynn Parry CB (eds) Surgical disorders of the peripheral nerves. Churchill Livingstone, New York, pp 209-233
13. Dodds SD, Wolfe SW (2000) Perinatal brachial plexus palsy. Curr Opin Pediatr 12:40-47

14. Nath RK, Paizi M (2007) Scapular deformity in obstetric brachial plexus palsy: a new finding. Surg Radiol Anat 29:133-140

15. Nath RK, Melcher SE, Paizi M (2006) Surgical correction of unsuccessful derotational humeral osteotomy in obstetric brachial plexus palsy: evidence of the significance of scapular deformity in the pathophysiology of the medial rotation contracture. J Brachial Plex Peripher Nerve Inj 1:9

16. Nath RK, Paizi M (2007) Improvement in abduction of the shoulder after reconstructive soft-tissue procedures in obstetric brachial plexus palsy. J Bone Joint Surg Br 89:620-626

17. Narakas AO (1993) Muscle transpositions in the shoulder and upper arm for sequelae of brachial plexus palsy. Clin Neurol Neurosurg 95(Suppl):S89-S91

18. Friedman RJ, Hawthorne KB, Genez BM (1992) The use of computerized tomography in the measurement of glenoid version. J Bone Joint Surg Am 74:1032-1037

19. Nath RK, Somasundaram C, Melcher SE, Bala M, Wentz MJ (2009) Arm rotated medially with supination - the ARMS variant: description of its surgical correction. BMC Musculoskelet Disord 10:32

20. Nath RK, Humphries AD (2008) Computed tomography of the shoulders in patients with obstetric brachial plexus injuries: a retrospective study. Ann Surg Innov Res 2:4

21. Moukoko D, Ezaki M, Wilkes D, Carter P (2004) Posterior shoulder dislocation in infants with neonatal brachial plexus palsy. J Bone Joint Surg (Am) 86-A:787-793

22. Rollnik JD, Hierner R, Schubert M, Shen ZL, Johannes S, Troger M, Wohlfarth K, Berger AC, Dengler R (2000) Botulinum toxin treatment of cocontractions after birth-related brachial plexus lesions. Neurology 55:112-114

23. Waters PM (2005) Update on management of pediatric brachial plexus palsy. J Pediatr Orthop B 14:233-244

24. Al-Qattan MM (2002) Rotation osteotomy of the humerus for Erb's palsy in children with humeral head deformity. J Hand Surg Am 27:479-483

25. Kirkos JM, Papadopoulos IA (1998) Late treatment of brachial plexus palsy secondary to birth injuries: rotational osteotomy of the proximal part of the humerus. J Bone Joint Surg Am 80:1477-1483

26. Egloff DV, Raffoul W, Bonnard C, Stalder J (1995) Palliative surgical procedures to restore shoulder function in obstetric brachial palsy. Critical analysis of Narakas' series. Hand Clin 11:597-606

27. Waters PM, Bae DS (2006) The effect of derotational humeral osteotomy on global shoulder function in brachial plexus birth palsy. J Bone Joint Surg Am 88:1035-1042

28. Pearl ML, Edgerton BW, Kazimiroff PA, Burchette RJ, Wong K (2006) Arthroscopic release and latissimus dorsi transfer for shoulder internal rotation contractures and glenohumeral deformity secondary to brachial plexus birth palsy. J Bone Joint Surg Am 88:564-574

29. Nath RK, Amrani A, Melcher SE, Eichhorn MG (2009) Triangle tilt surgery in an older pediatric patient with obstetric brachial plexus injury. ePlasty 9:e26

30. Waters PM (1997) Obstetric brachial plexus injuries: evaluation and management. J Am Acad Orthop Surg 5:205-214

31. Hui JH, Torode IP (2003) Changing glenoid version after open reduction of shoulders in children with obstetric brachial plexus palsy. J Pediatr Orthop 23:109-113 